\title{
Lower limb tri-joint synchrony during running gait: A longitudinal age-based study
}

Authors: Ceri Diss ${ }^{\mathrm{a}}$, Domenico Vicinanza ${ }^{\mathrm{b}}$, Lee $\mathrm{Smith}^{\mathrm{c}}$, Genevieve K. R. Williams ${ }^{\mathrm{d}}$

aDepartment of Life Sciences, University of Roehampton, London, UK,

email:c.diss@roehampton.ac.uk

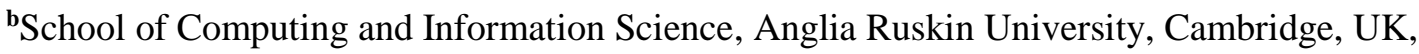

email: domenico.vicinanza@anglia.ac.uk

${ }^{\mathrm{c} C a m b r i d g e}$ Centre for Sport and Exercise Sciences, Anglia Ruskin University, Cambridge, UK, email: lee.smith@anglia.ac.uk

${ }^{d}$ School of Sport and Health Sciences, University of Exeter, UK,

email: g.k.r.williams@exeter.ac.uk

Corresponding Author Details:

Dr Genevieve Williams

Email: g.k.r.williams@exeter.ac.uk

Address: School of Sport and Health Sciences, University of Exeter, UK.

Acknowledgements:

Marianne Gittoes, David Kerwin, Richard Tong, George Weeks 


\begin{abstract}
Biomechanical research exploring the age-based mechanics of running gait can provide valuable insight into the reported decline in master endurance running performance. However, few studies have shown consistent biomechanical differences in the gait of trained distance runners compared to their younger counterparts. It might be that differences occur in the interaction between joints. The aim was to explore the differences in in tri-joint synchrony of the lower limb, quantified through Cluster Phase analysis, of runners at 50 years of age compared to seven years later. Cluster Phase analysis was used to examine changes in synchrony between 3 joints of the lower limb during the stance phase of running. Ten male, endurance-trained athletes M50 (age $=53.54 \pm 2.56$ years, mass $=$ $71.05 \pm 7.92 \mathrm{~kg}$ ) participated in the study and returned after seven years M57 (age $=60.49 \pm 2.56$ years, mass $=69.08 \pm 8.23 \mathrm{~kg}$ ). Lower limb kinematics (Vicon, $120 \mathrm{~Hz}$ ) and ground reaction forces (Kistler, $1080 \mathrm{~Hz}$ ) were collected as participants performed multiple trials at a horizontal running velocity $=$ $3.83 \pm 0.40 \mathrm{~m} \cdot \mathrm{s}^{-1}$ over the force plate. Significant increase $(31 \%)$ in rate of force development in the absorption phase, and significantly reduced sagittal plane knee joint range of motion $\left(30.50 \mathrm{v} 23.68^{\circ}\right)$ were found following the seven years of ageing. No further discrete single joint measures were significantly different between M50 and M57. Joint synchrony between the hip, knee and ankle was significantly higher at M57 compared to M50 during the absorption phase of stance. The force attenuation strategy is compromised after seven years of ageing, which is associated with more synchronous movements in the lower limb joints. Increased joint synchrony as a function of age could be a mechanism associated with this key injury provoking phase of running gait.
\end{abstract}

\title{
Word count: 286
}

Key words: Cluster Phase, Dynamical Systems Theory, Coordination, Lower Body Kinematics 


\section{Highlights}

- A seven-year longitudinal study of running mechanics for athletes

- Cluster Phase quantified synchrony between three lower limb joints during stance

- Rate of vertical force development increased, while knee joint range of motion decreased

- Joint synchrony during the absorption phase is higher after 7 years of ageing

- With age force attenuation is compromised and lower limb-joint synchrony increases 


\subsection{Introduction}

Biomechanical research exploring the age-based mechanics of running gait can provide valuable insight into the reported decline in master endurance running performance (Tarpenning, Hamilton-Wessler, Wiswell, \& Hawking, 2004). To date, knee joint kinematics, ankle joint stiffness and impact forces have been identified as key biomechanical variables of running gait that change as a function of age (Bobbert, Schamhardt \& Nigg, 1991; Bus, 2003; Karamanidis, Arampatzis, \& Mademli, 2008; Fukuchi \& Duarte, 2008; DeVita, Fellin, Seay, Ip, Stavro, \& Messier, 2016;). For example, reduced range of motion of the knee joint during ground contact is suggested as a common characteristic of ageing runners (Fukuchi \& Duarte, 2008), and it has been attributed to a decrease in strength of the tricep surae and quadriceps femoris muscle-tendon units (Bus, 2003). During the stance phase of running, no age-based differences in the sagittal plane ankle range of motion have been observed in runners aged 55-65 years, suggesting that such measures do not contribute to the reported increases in ankle stiffness (DeVita et al., 2016). However, DeVita et al. (2016) reported that hip and knee mechanics were unaffected by age, while, mechanical adaptations at the ankle joint did exist (DeVita et al., 2016; Bobbert et al., 1991). Therefore, there is need for further examination of age-based changes in running mechanics. While the majority of research examining changes in running gait with ageing has been cross sectional in nature (c.f. Bobbert et al., 1991; Taunton, Ryan, Clement, 2002; Lilley, Dixon, \& Stiles, 2011), a longitudinal study design might be more powerful, since it considers individual responses beyond a single time point in a repeated measures design.

Since it is likely that age induces changes in the kinematics of masters' running gait, but few studies have shown consistent biomechanical differences (Bobbert et al., 1991; De Vita et al., 2016), it might be that differences occur in the interaction between joints. A major focus of the dynamical systems approach to motor control is to understand how the components within a system (e.g., joint space degrees of freedom) become coordinated in order to effectively and efficiently meet task demands (Kugler, Kelso, \& Turvey, 1980; Newell, 1985; Turvey, 1990; Kelso, 1995). In this view, the phase relations between the mechanical degrees of freedom of the lower extremities during running (intra-limb coordination) have been investigated in walking and 
running (Diedrich \&Warren, 1998; Li, van den Bogert, Caldwell, van Emmerik, \& Hamill, 1999), in relation to lower limb injuries (Hamill, van Emmerik, Heiderscheit, \& Li, 1999), in the investigation of lower limb segment motions (Stergiou, Bates, \& James, 1997; Lamoth, Beek, \& Meijer, 2002; Takabayashi, Edama, Nakamura, Yokoyama, Kanaya, \& Kubo, 2017), specific gait conditions including obstacles and over-ground v treadmills (Burgess-Limerick, Neal \& Abernethy, 1992; Clark \& Phillips, 1993; van Emmerik, \& Wagenaar, 1996; Stergiou, Jensen, Bates, Scholten, \& Tzetzis, 2001; Ferber, Davis, \& Williams, 2005; Chiu, Chang, \& Chou, 2015), and in young and elderly populations (Byrne, Stergiou, Blanke, Houser, Kurz, \& Hageman, 2002; Chiu \& Chou, 2012; Chiu \& Chou, 2013). More specifically, differences in lower limb coordination during gait as a function of age have been explored (Byrne at al., 2002; Chiu \& Chou, 2012; Chiu \& Chou, 2013). Byrne et al. (2002) reported a more in-phase relative phase between the shank and thigh during the braking phase of walking for older adults, compared to younger adults. If also corroborated for running, this finding has potential implications for injury. Stergiou, Jensen, Bates, Scholten, \& Tzetzis (2001) in fact suggested more in-phase coordination of the lower-extremity segments may limit the impact-absorbing capacity during the stance phase of running and be associated with an increase in collision forces. The coordinated, sequential movements of the lower extremities contribute to absorbing the impact force by increasing the time and displacement over which breaking of acceleration occurs. Finally, Stergiou, Scholten, Jensen, \& Blanke (2001) showed that a relatively out-of-phase motion during the stance phase might be associated with the desire to reduce the landing load. Therefore, there is need to explore the interaction between joints, where we might expect more in-phase or synchronous relations between the lower extremity joints as a function of ageing.

The majority of studies have reported coordination variability, with only some reference to the underlying coordinative structure. For example, coordination variability has been explored in line with injury and ageing. In recent literature, Wang, Gu, Wang, Siao, \& Chen (2018) showed that lower deviation phase between hip and knee flexion/extension was associated with higher impact forces during the absorption phase of running. This is in line with the findings of Goldberger (1991) and van Emmerik, Hamill, \& McDermott (2005) who suggested that constraining 
movement (characterized by lower movement variability and fewer movement solutions) may not be conducive to absorbing the collision load, and Hamill, van Emmerik, Heiderscheit, \& Li (1999) who suggested that greater variability was considered to be functional, while less variable inter-joint coordination might induce cartilage tissue to repeatedly experience greater stress associated with patellofemoral pain. Boyer, Silvernail, \& Hamill (2016) reported non-equivalent changes in the coordination variability of different lower limb segment couplings for men and women of different ages, while Silvernail, Boyer, Rohr, Brüggemann, \& Hamill (2015) did not find significant difference in coordination variability of the lower extremity with age; therefore, findings are not unequivocal. It is the focus of this work however to explore changes in the coordination profiles.

However, while these studies have enabled a better understanding of the coordination of our biomechanical system during gait using bivariate methods such as relative phase, continuous relative phase, vector coding, spectral coherence, cross-correlation and cross-recurrence analysis (Richardson, Garcia, Frank, Gregor, \& Marsh, 2012; van Emmerik, Ducharme, Amado, \& Hamill, 2016), they are still limited to exploring the coordination and coordination variability between two mechanical degrees of freedom. In order to capture coordination of a greater number of system components, a number of techniques have been explored in recent literature. For example, to capture the collective state of the system, Segers, Aerts, Lemoir, \& De Clercq (2007) described the phase relations between two biomechanically relevant global variables: kinetic energy and gravitational potential energy during walking and running. Thus, in capturing global variables, they were able to capture more information from the system than only looking at the phases between two joints or segments. Alternatively, embracing the multiple degree of freedom problem, statistical methods such as Principal Component Analysis have been used to reduce the dimensionality of mechanical degrees of freedom for all body segments (Daffertshofer, Lamoth, Meijer, \& Beek, 2004; Lamoth, Daffertshofer, Huys, \& Beek, 2009), increasing our understanding of the coordination involved in gait patterns. The Uncontrolled Manifold hypothesis has been tested in relation to gait stability (Papi, Rowe, \& Pomeroy, 2015) and hopping performance (Yen \& Chang, 2010). More recently, Williams \& Vicinanza (2017) 
presented a method to consider the relations between multiple oscillators using frequency

87 decomposition. To date however, no studies have investigated coordination in the three key joints that make up the lower limb.

Our approach to study the coordination (as simultaneous synchrony) between three joints is adapting the Cluster Phase method proposed by Frank \& Richardson (2010). The method is based on the Kuramoto order parameter (Kuramoto, 1984; Kuramoto 1989), which has been previously used to study synchronization of many-body systems in life (Walker, 1969, for cricket synchronization), social sciences (Néda, Ravasz, Brechet, Vicsek, \& Barabási, 2000 and 2000b, for synchronized applause) and sports sciences (Duarte, Araújo, Correia, Davids, Marques, \& Richardson, 2013; for synchronization among players in a football team). Frank \& Richardson (2010) adapted and successfully showed the applicability of this method to examine synchronization of a smaller number of oscillators. Specific measures of individual and whole group synchrony obtained with this method were able to distinguish intentional from chance level coordination tendencies between the rocking of six chairs (Frank \& Richardson, 2010), suggesting that Cluster Phase might be a viable technique to explore the synchrony present in limb and whole body degrees of freedom.

101 Aim

102 The aim of this paper was to quantify tri-joint coordination through Cluster Phase analysis, to examine changes in the age-based synchronization in the lower limb joints during running, following seven years of ageing.

\subsection{Methods}

\section{$106 \quad 2.1$ Participants and Procedures}

107 Ten male endurance-trained athletes (age $=53.54 \pm 2.56$ years, mass $=71.05 \pm 7.92 \mathrm{~kg}$ ) volunteered to participate in the study and returned to the study seven years later (age $=$ $60.49 \pm 2.56$ years, mass $=69.08 \pm 8.23 \mathrm{~kg}$ ). M50 defined the initial data collection and M57 the data collection seven years later. The criterion for inclusion in the study required the athletes, at the time of data collection, to: be injury free, participate in a minimum of five running-based

112 training sessions per week (two of which were at an intensity that exceeded the lactate threshold), 113 have a personal best time for $10 \mathrm{~km}$ of less than 40 minutes, recently finish in the top twenty 
114 positions in the regional county championships. All athletes provided written informed consent,

115 and ethical approval for the data collection protocol was gained from the host University's Ethics

116 Board prior to study onset.

\section{$117 \quad$ 2.2 Data Collection}

118 Passive markers (14mm in diameter) were placed at precise anatomical landmarks and

119 anthropometric measurements were recorded in accordance with the lower body Plug-in-Gait model (Vicon ${ }^{\mathrm{TM}}$, Oxford). Following a familiarisation period participants performed multiple running trials at a standardised horizontal velocity $=3.83 \pm 0.40 \mathrm{~m} \cdot \mathrm{s}^{-1}$ whilst making right footground contact with a force plate situated $13 \mathrm{~m}$ along the $20 \mathrm{~m}$ runway. Participants typically performed 20 running trials which ensured at least six trials were successful for further analysis i.e. a clean foot strike and correct running velocity. Three-dimensional coordinate (sample rate: $120 \mathrm{~Hz}$ ) data of the passive markers were collected using a 12 camera Vicon system (Vicon ${ }^{\mathrm{TM}}$, Oxford) synchronized with a Kistler force plate (Kistler ${ }^{\mathrm{TM}}$, Switzerland, 9281C; sample rate: $1080 \mathrm{~Hz}$ ). The protocol and data collection was replicated seven years later.

\subsection{Data Processing}

129 Non-linear transformation was used to reconstruct the 3D coordinate data of each marker. The respective time histories were smoothed using Woltring's cross-validated quintic spline with the mean square error noise tolerance level set to $15 \mathrm{~mm}^{2}$ from which the joint centres of the lower body were determined. Sagittal plane hip, knee and ankle flexion/extension angles were determined using vector defined segments.

134 Stance phase angular kinematics and ground contact forces of each running trial were analysed and defined between the instants of initial ground contact $(\mathrm{Fz}>8 \mathrm{~N})$ and toe off $(\mathrm{Fz}<8 \mathrm{~N})$ with the force plate. Stance phase was divided into two sub-phases: absorption and propulsion, which were distinguished by the time when the horizontal ground contact force $=0 \mathrm{~N}$. The hip, knee and ankle joint flexion angles were reported at initial ground contact. Joint ranges of motion (ROM) were reported during the absorption phase. The peak vertical impact force, rate of

140 vertical force development in the absorption phase and the vertical force at the time of the transition

141 between the absorption and propulsion phase (normalised to body weight) were examined. Individual 
142 stance phase waveform profiles of the joint angle measures were interpolated to 101 points using a

143 cubic spline (MathCad 13, Adept Scientific). Average of all stance phase measures were calculated

144 for each athlete from six athlete-specific trials for both data collection sessions. The group means

145 (standard deviation) were then determined.

$146 \quad$ 2.3.1 Cluster Phase

147 The cluster phase method used to assess the synchrony between three joints is Frank and Richardson's

148 (Frank \& Richardson, 2010)) adaptation of the Kuramoto order parameter method (Kuramoto, 1984).

149 Frank and Richardson (Frank \& Richardson, 2010) tailored the Kuramoto model, typically defined for

150 a very large number of oscillatory units (thermodynamic limit, Kuramoto \& Nishikawa, 1987), in

151 order to work with systems with a small number of oscillators.

152 For each of the three joints time-series, $x_{\text {hip }}\left(t_{i}\right), x_{\text {knee }}\left(t_{i}\right), x_{\text {ankle }}\left(t_{i}\right)$, where $t_{i}, i=1, \ldots, N$ are the

153 time steps, the phase time-series in radians $[-\pi, \pi]$ for $\theta_{\text {hip }}, \theta_{\text {knee }}, \theta_{\text {ankle }}$ was calculated, using the

154 Hilbert transform (Kuramoto \& Nishikawa, 1987; Strogatz, 2000). Then, from the phase time-series

155 we calculated the cluster phase as follows:

$156 \dot{q}\left(t_{i}\right)=\frac{1}{3} \sum_{i=1}^{N}\left(\exp \left(i \theta_{\text {hip }}\left(t_{i}\right)\right)+\exp \left(i \theta_{\text {knee }}\left(t_{i}\right)\right)+\exp \left(i \theta_{\text {ankle }}\left(t_{i}\right)\right)\right)$

157 and

$158 q\left(t_{i}\right)=\operatorname{atan} 2\left(\dot{q}\left(t_{i}\right)\right)$

159 where $i=\sqrt{-1}$ (when not used as a time step index), and $q\left(t_{i}\right)$ and $q\left(t_{i}\right)$ are the resulting group or

160 cluster phase in complex and radian $[-\pi, \pi]$ forms, respectively.

161 The cluster phase calculated is a description of the global synchrony of the three joints. Based on the

162 global cluster phase $q\left(t_{i}\right)$, the relative phases for the individual joints, $\phi_{\text {hip }}\left(t_{i}\right)$,

$163 \phi_{\text {knee }}\left(t_{i}\right), \phi_{\text {ankle }}\left(t_{i}\right)$, can be calculated as:

164

$\phi_{\text {hip,knee,ankle }}\left(t_{i}\right)=\theta_{\text {hip,knee, ankle }}\left(t_{i}\right)-q\left(t_{i}\right)$ 
165 Where $\phi_{\text {hip,knee, ankle }}\left(t_{i}\right)=\theta_{\text {hip,knee,ankle }}\left(t_{i}\right)-q\left(t_{i}\right)$ is the compact form for the three equations: $\phi_{\text {hip }}\left(t_{i}\right)=\theta_{\text {hip }}\left(t_{i}\right)-q\left(t_{i}\right), \phi_{\text {knee }}\left(t_{i}\right)=\theta_{\text {knee }}\left(t_{i}\right)-q\left(t_{i}\right)$ and $\phi_{\text {ankle }}\left(t_{i}\right)=\theta_{\text {ankle }}\left(t_{i}\right)-q\left(t_{i}\right)$.

167 As a next step, mean relative phase $\bar{\phi}$ and the degree of synchrony $\rho$ for every joint with respect to the cluster (group) behaviour is calculated from:

169

$\overline{\hat{\phi}_{\text {hlp }}}=\frac{1}{N} \sum_{i=1}^{N} \exp \left(i \phi_{\text {hip }}\left(t_{i}\right)\right)$

172 and

$173 \overline{\phi_{\text {hıp,knee,ankle }}}=\operatorname{atan} 2\left(\overline{\hat{\phi}_{\text {hıp,knee,ankle }}}\right)$

$174 \quad \rho_{\text {hip,knee, ankle }}=\overline{\left|\dot{\phi}_{\text {hıp,knee, ankle }}\right|}$

175 where $\bar{\phi}$ and $\bar{\phi}$ is the mean relative phase in complex and radian $[-\pi, \pi]$ forms, and $\rho \in[0,1]$.

176 As mentioned in Richardson et al. (2012), if $\rho=1$ the movement is in complete synchrony with the

177 group (i.e., the phase of the movement at any time step is equivalent to the group phase shifted by a constant phase). If $\rho=0$ the movement is completely unsynchronized to the group.

179 Finally, the degree of synchrony of the three joints to the group as a whole $\rho_{\text {group }}$ at every time step $t_{i}$ is defined by:

183 It is worth noting that $\rho_{\text {group }, i}$ provides a continuous measurement ( $i$ is the time index) of the group

184 synchrony. In addition, $\rho_{\text {group }, i} \in[0,1]$ and from which the average degree to group synchrony was calculated as: 
187 Note that $\rho_{\text {group }}$ provides a single measure of group synchrony for the experiment (behavioural

188 period or trial) and, again, the closer to 1 the value of $\rho_{\text {group }, i}$ and $\rho_{\text {group }}$ larger the degree of group 189 synchrony.

190

191

192

193

194

195

\subsection{Data analysis}

The Shapiro-Wilk statistical test for normal distribution revealed that all measures were normally distributed. Statistical analysis has been conducted using multivariate analysis of variance (MANOVA), to account for several dependent variables (flexion values, impact peak, rate of force development, vertical force, joint and mean synchrony values)..

\subsubsection{Statistical Parametric Mapping}

Statistical parametric mapping (SPM) technique with paired t-test was used to examine the differences in the waveform joint angle data for M50 and M57. SPM was designed especially for continuous field analysis (Friston, Ashburner, Kiebel, Nichols, \& Penny, 2007) and constructs images that lie in the original, biomechanically meaningful sampling space (Pataky, 2010). Opensource one-dimensional package for Matlab (spm1d version M.0.3.1 (2015.08.28)) was used in the analysis and the scalar test statistic $\operatorname{SPM}\{t\}$ was computed at each point in the time series as described previously by Robinson, Vanrenterghem \& Pataky (2015).

\subsection{Results}

\subsection{Joint angles and vertical force}

There was no significant differences between the M50 and M57 discrete measures of joint flexion angles at touch down and ROM in the absorption phase except for a significantly reduced knee joint ROM for M57 compared to M50 (Table 1;, $p=0.006$, effect size $=0.35$ ). Figure 1 illustrates the joint angle waveforms throughout the stance phase for the hip, knee and ankle. SPM found no significant differences between angles at M50 and M57 for each \% during the stance phase. Vertical impact peak force increased, with an average of $21 \%$, following a seven-year period of ageing, although the difference was not significant $(p=0.454)$. Rate of vertical force 
212 development in the absorption phase significantly increased for all participants, by average a $31 \%$

213 from M50 to M57, (Table 1; $p=0.025$, effect size $=0.23$ ).

214

215

216

217

218

219

220

221

222

223

224

225

226

227

228

229

230

231

232

233

234

235

236

237
Insert Table 1 around here Insert Figure 1 around here

\subsection{Joint synchronization}

\subsubsection{Average degree of synchrony of the group (joint synchrony)}

Average joint synchrony $\rho_{\text {group }}$ (Eq. 10) measures the presence and magnitude of the tri-joint synchrony. An example time series of average synchrony is depicted in Figure 2. It is evident that for the M50 years data, average synchrony is lower during the absorption phase of stance, compared to M57 years data.

Average joint synchrony $\rho_{\text {group }}$ across the entire stance or the propulsion phase was not significantly different between M50 and M57 (Figure 3; Table 1). Average joint synchrony across the absorption phase was significantly different (Table $1 ; p=0.008$, effect size $=0.34$ ) between M50 compared to M57 years.

\subsubsection{Average degree of synchrony of individual joints}

The average degree of synchrony of the individual joints $\rho_{\text {hip,knee,ankle }}$ (Eq. 8) quantifies the average degree to which each individual joint was synchronised to the movements of the three joints as a whole. An example time series of each joint's synchrony during the stance phase at M50 and M57 is depicted in Figure 4. The average degree of synchrony for the absorption phase was significantly higher for the hip (Table $1 ; p=0.039$, effect size $=0.31$ ), knee (Table $1 ; p=$ 0.005 , effect size $=0.39)$ and the ankle (Table $1 ; p=0.015$, effect size $=0.48)$ between M50 and 
M57. There was no significant difference in average degree of synchrony for the propulsion

239 phase the hip $(p=0.225)$, knee $(p=0.219)$, or ankle $(p=0.324)$ between M50 and M57 years.

\subsection{Discussion}

244 The current paper examined changes in the age-based synchronization in the lower limb joints

245 during running, following seven years of ageing, to further understand how running technique

246 changes as a function of age. To the authors' knowledge this is the first longitudinal research of

247 changes in the gait kinematics and kinetics of competitive endurance runners. Discrete and waveform analysis examined the changes in lower body kinematics and ground reaction forces during the stance phase of running. To further understanding of the organisation of the lower limb movements, Cluster Phase was used to examine changes in tri-joint synchrony. Rate of force development, and joint synchrony during the absorption phase increased at M57 compared to

252 M50. In particular, rate of vertical force development in the absorption phase was significantly higher at M57, by an average of $31 \%$. Peak vertical impact force, even though not significantly higher at M57, increased by an average of $21 \%$, compared to M50. To date, there is debate in the literature as to whether this vertical force increases, decreases, or does not significantly change with age (Power, Dalton, Behm, Vandervoort, Doherty \& Rice, 2010; Kline \& Williams, 2015; Diss,

258 Weeks, Gittoes, Tong, \& Kerwin, 2015; DeVita et al., 2016). However, these previous studies were based on cross sectional designs and the results are therefore confounded by inter-individual differences in and between, groups. Based on the longitudinal design used here, both the rate of force and peak measures suggest a required augmentation in runners' ability to attenuate vertical forces or improve muscle activation prior to impact with the ground, following a period of ageing.

264 Analysis of typically reported discrete sagittal plane joint angles found only the knee joint ROM measure to be significantly different after a seven-year period of ageing. It is acknowledged that 
significant decreases in masters endurance running performance occur after 50 years of age (De

267 Vita et al., 2016), however it is suggested that exposure to the rigors of competitive running

268 provides a unique landscape for the changes that occur (Power et al., 2010). In agreement with previous research (Fukuchi \& Duarte, 2008; Lilley et al., 2011; De Vita et al., 2016) knee ROM in the absorption phase was shown to decrease with age. Reduced knee kinematics have been associated with reductions in strength of the triceps surae and quadriceps femoris muscle-tendon units (De Vita et al., 2016) and suggest that the force attenuation strategy is altered, which is

273 concerning due to this injury provoking absorption phase of stance.

274 This is the first application of the Cluster Phase method to exploring the relations between multiple joint actions during human movement. Average synchrony in the current analysis ranged between 0.70 and 0.99 , suggesting that the current system represents a unit with synchrony that is in line with that of synchronising of rocking chairs reported in (Frank \& Richardson, 2010) and the synchrony among players in a football team (Duarte et al., 2013).

279 Tri-joint synchrony significantly increased in the absorption phase of stance at M57, compared to 280 M50, demonstrating that the fundamental biomechanical interaction of the joints underpinning the absorption of force has changed. Specifically, increased synchrony indicated that the hip, knee and ankle are working more as a single unit where the timings are more similar and phases coherent. The mechanical constraint of increased synchrony that appears to have arisen as a consequence of ageing could be associated with loss of complexity (Lipsitz \& Goldberger, 1992), where the three joints are operating as a single, more synchronous unit during ageing. Moreover, the increase in vertical ground reaction force variables suggests that this increase in lower limb joint synchrony is a less functional solution, and thus further research might explore this proposition.

Previous research exploring coordination in gait has reported more in-phase coupling between the shank and thigh during the breaking phase of walking for older adults compared to younger adults (Byrne et al., 2002). Stergiou, Jensen, et al. (2001) suggested more in-phase coordination of the lower-extremity segments may limit the impact-absorbing capacity during stance phase of running, and be associated with an increase in collision forces. Stergiou, Scholten, et al. (2001) 
294 showed that a relatively out-of-phase motion during the stance phase might be associated with the

295 desire to reduce the landing load. This is in line with the current findings and suggests that

296 measures of coordination and synchrony could be key to understanding changes in gait with age,

297 in line with both the theories and motor control and biomechanics and injury. Specifically, it is

298 suggested that the coordinated, sequential movements of the lower extremities contribute to

299 absorbing the impact force to a greater extent than those that are more synchronised, by

300 increasing the time and displacement over which breaking of acceleration occurs.

301

$302 \quad 5.0$ Conclusion

303 An increase in tri-joint synchrony in the absorption phase of stance after seven years indicates that the

304 hip, knee and ankle are working more as a single unit where the timings are more similar and phases

305 more coherent. The mechanical constraint of increased synchrony that appears to have arisen as a

306 consequence of ageing could be associated with the increase in vertical ground reaction force

307 variables.

308 The results from this study suggest that the cluster phase method can be used to identify coordination

309 changes in three joints during running as a function of changing biological constraints.

310 Future work could examine whether there is limited adaptability in this synchronization in response to

311 perturbations in the running surface with ageing, for example. It might also be explored whether

312 increased synchrony is a characteristic of aged gait and movement per se. 


\section{Conflict of interest statement}

There are no conflicts of interest associated with this work. Neither Dr C. Diss, D. Vicinanza, L.

Smith or G. Williams have any financial or personal relationships with other people or organisations that could inappropriately influence (bias) this work. 


\section{References}

Bobbert, M. F., Schamhardt, H. C., \& Nigg, B. M. Calculation of vertical ground reaction force estimates during running from positional data. Journal of Biomechanics, 24, 1095-1105.

Boyer K., Silvernail, J.F., \& Hamill, J. (2016). Age and sex influences on running mechanics and coordination variability. Journal of Sports Sciences, 35, 2225-2231. DOI:

$10.1080 / 02640414.2016 .1265139$

Burgess-Limerick, R., Neal, R. J., \& Abernethy, B. (1992). Relative timing and phase angle invariance in stair-climbing. Quarterly Journal of Experimental Psychology, 44A, 705-722.

Bus, S. A. (2003). Ground reaction forces and kinematics in distance running in older-aged men. Medicine Science and Sports Exercise, 35, 1167-1175.

Byrne, J. E., Stergiou, N., Blanke, D., Houser, J. J., Kurz, M. J., \& Hageman, P. A. (2002).

Comparison of gait patterns between young and elderly women: an examination of coordination. Perceptual and Motor Skills, 94(1), 265-80.

Chiu, S. L., \& Chou, L. S. (2012). Effect of walking speed on inter-joint coordination differs between young and elderly adults. Journal of Biomechanics, 45(2), 275-80.

Chiu, S. L., \& Chou, L. S. (2013). Variability in inter-joint coordination during walking of elderly adults and its association with clinical balance measures. Clinical Biomechanics, 28(4), 454-8. Chiu, S. L., Chang, C. C., \& Chou, L. S. (2015). Inter-joint coordination of overground versus treadmill walking in young adults. Gait and Posture, 41(1), 316-8.

Clark, J. E., \& Phillips, S. J. (1993). A longitudinal study of intralimb coordination in the first year of independent walking: a dynamical systems analysis. Child Development, 64(4), 1143-57.

Daffertshofer A., Lamoth, C. J., Meijer, O. G., \& Beek, P. J. (2004). Principal components analysis in studying coordination and variability: A tutorial. Clinical Biomechanics, 19(4), 415-428.

DeVita, P., Fellin R. E., Seay, J. F., Ip, E., Stavro, N., \& Messier, S. P. (2016). The relationships between age and running biomechanics. Medicine and Science in Sports Exercise, 48(1), 98-106. Diedrich, F. J., \& Warren, Jr. W. H. (1998). The dynamics of gait transitions: Effects of grade and load. Journal of Motor Behavior, 30(1), 60-78. 
Diss, C. E., Weeks, G., Gittoes, M. J., Tong, R. J., \& Kerwin, D. G. (2015). Age-based biomechanics of running gait: A longitudinal study. Gait and Posture, 42, S72-3.

Duarte, R., Araújo, D., Correia, V., Davids, K., Marques, P., \& Richardson, M. J. (2013). Competing together: Assessing the dynamics of team-team and player-team synchrony in professional association football. Human Movement Science, 32(4), 555-66.

Ferber, R., Davis, I. M., \& Williams, D. S. (2005). Effect of foot orthotics on rearfoot and tibia joint coupling patterns and variability. Journal of Biomechanics, 38(3), 477-83.

Frank, T. D., \& Richardson, M. J. (2010). On a test statistic for the Kuramoto order parameter of synchronization: An illustration for group synchronization during rocking chairs. Physica D:

Nonlinear Phenomena, 239(23-24), 2084-92.

Friston, K. J, Ashburner, J. T, Kiebel, S. J, Nichols, T. E, \& Penny, W. D. (2007). Statistical parametric mapping: the analysis of functional brain images. London: Elsevier.

Fukuchi, R. K., \& Duarte, M. (2008). Comparison of three-dimensional lower extremity running kinematics of young adult and elderly runners. Journal of Sport Science, 26(13), 1447-54.

Goldberger, A. L. (1991). Is the normal heartbeat chaotic or homeostatic? Physiology 6(2), 87-91.

Hamill, J., van Emmerik, R. E., Heiderscheit, B. C., \& Li, L. (1999). A dynamical systems approach to lower extremity running injuries. Clinical Biomechanics, 14(5), 297-308.

Hamill, J., van Emmerik, R. E., Heiderscheit, B. C., Li, L. (1999). A dynamical systems approach to lower extremity running injuries. Clinical Biomechanics, 14(5), 297-308. DOI:10.1016/S0268-

Karamanidis, K., Arampatzis, A., \& Mademli, L. (2008). Age-related deficit in dynamic stability control after forward falls is affected by muscle strength and tendon stiffness. Journal of Electromyography and Kinesiology, 18(6), 980-9.

Kelso, J. A. S. (1995). Dynamic patterns: The self organization of brain and behavior. Cambridge, MA: The MIT Press.

Kline, P. W., \& Williams, D. S. (2015). Effect of normal ageing on lower extremity loading and coordination during running in males and females. International Journal of Sports Physical Therapy, 10(6), 901-909. 
Kugler, P. N., Kelso, J. A. S., \& Turvey, M. T. (1980). On the concept of coordinative structures as dissipative structures. I. Theoretical lines of 191 convergence. G. E. Stelmach: Tutorials in motor behavior. Amsterdam: North-Holland.

Kuramoto, Y. (1984). Chemical Oscillations, Waves, and Turbulence. Berlin: Springer.

Kuramoto, Y. (1989). Cooperative Dynamics in Complex Physical Systems. Berlin: Springer.

Kuramoto, Y., \& Nishikawa, I. (1987). Statistical macrodynamics of large dynamical systems case of a phase transition in oscillator communities. Journal of Statistical Physiology, 49, 569.

Lamoth, C. J, Daffertshofer, A., Huys, R., \& Beek, P. J. (2009). Steady and transient coordination structures in human walking and running. Human Movement Science, 28, 371-386.

Lamoth, C. J., Beek, P. J., \& Meijer, O. G. (2002). Pelvis-thorax coordination in the transverse plane during gait. Gait and Posture, 16(2), 101-14.

Li, L., van den Bogert, E. C., Caldwell, G. E., van Emmerik, R. E., \& Hamill, J. (1999). Coordination patterns of walking and running at similar speed and stride frequency. Human Movement Science, $18(1), 67-85$.

Lilley, K., Dixon, S., \& Stiles, V. (2011). A biomechanical comparison of the running gait of mature and young females. Gait and Posture, 33(3), 496-500.

Lipsitz, L. A., \& Goldberger, A. L. (2002). Loss of complexity and aging. Jama, 267(13), 1806-1809. McClay, I., \& Manal, K. (1997). Coupling parameters in runners with normal and excessive pronation. Journal of Applied Biomechanics, 13(1), 109-24.

Nawoczenski, D. A., Cook, T. M., \& Saltzman C. L. (1995). The effect of foot orthotics on threedimensional kinematics of the leg and rearfoot during running. Journal of Orthopaedic \& Sports Physical Therapy, 21(6), 317-27.

Néda, Z., Ravasz, E., Brechet, Y., Vicsek, T., \& Barabási A. L. (2000). Physics of rhythmic applause. Physics Reviews, E61, 6987-6992,

Néda, Z., Ravasz, E., Brechet, Y., Vicsek, T., \& Barabási, A. L. (2000). The sound of many hands clapping. Nature, 403, 849-850.

Newell, K. M. (1985). Coordination, Control and Skill. In Advances in Psychology: North-Holland; 27: $295-317$. 
Papi, E., Rowe, P. J., Pomeroy, \& V. M. (2015). Analysis of gait within the uncontrolled manifold hypothesis: Stabilisation of the centre of mass during gait. Journal of Biomechanics, 48(2), 324-331. Pataky, T. C. (2010). Generalized n-dimensional biomechanical field analysis using statistical parametric mapping. Journal of Biomechanics, 43(10), 1976-82.

Power, G. A, Dalton, B. H., Behm, D. G., Vandervoort, A. A., Doherty, T. J., \& Rice, C. L. (2010). Motor unit number estimates in masters runners: use it or lose it? Medicine and Science in Sports Exercise, 42, 1644-1650. doi: 10.1249/MSS.0b013e3181d6f9e9

Richardson, M., Garcia, R. L., Frank, T. D., Gregor, M., \& Marsh, K. L. (2012). Measuring group synchrony: a cluster-phase method for analyzing multivariate movement time-series. Frontiers in Physics, 3, 405.

Robinson, M. A, Vanrenterghem, J., \& Pataky, T. C. (2015). Statistical Parametric Mapping (SPM) for alpha-based statistical analyses of multi-muscle EMG time-series. Journal of Electromyography and Kinesiology, 25(1), 14-9.

Segers, V., Aerts, P., Lemoir, M., \& De Clercq, D. (2007). Dynamics of the body centre of mass during actual acceleration across transition speeds. Journal of Experimental Biology, 210, 578-585.

Silvernail, J.F., Boyer K., Rohr, E., Brüggemann, G.P., \& Hamill, J. (2015). Running Mechanics and Variability with Aging. Medicine and Science in Sports and Exercise, 42, 2175-2180. DOI: $\underline{10.1249 / \mathrm{MSS} .0000000000000633}$

Stergiou, N., Bates, B. T., \& James, S. L. (1997). Asynchrony between subtalar and knee joint function during running. Gait \& Posture, 6(3), 177-185.

Stergiou, N., Jensen, J. L., Bates, B. T., Scholten, S. D., \& Tzetzis, G. A. (2001). Dynamical systems investigation of lower extremity coordination during running over obstacles. Clinical Biomechanics, 16(3), 213-221. DOI:10.1016/S0268-0033(00)00090

Stergiou, N., Jensen, J. L., Bates, B. T., Scholten, S.D., \& Tzetzis, G. (2001). A dynamical systems investigation of lower extremity coordination during running over obstacles. Clinical Biomechanics, 16(3), 213-21. 
Stergiou, N., Scholten, S. D., Jensen, J. L., \& Blanke, D. (2001). Intralimb coordination following obstacle clearance during running: the effect of obstacle height. Gait and Posture, 13(3), 210-220. DOI:10.1016/S0966-6362(00)00101-6

Strogatz, S. H. (2000). From Kuramototo Crawford: exploring the onset of synchronization in populations of coupled nonlinear oscillators. Physica D. 143, 1-20.

Takabayashi, T., Edama, M., Nakamura, E., Yokoyama, E., Kanaya, C., \& Kubo, M. (2017). Coordination among the rearfoot, midfoot, and forefoot during walking. Journal of Foot and Ankle Research, 10(1), 42.

Tarpenning K. M., Hamilton-Wessler M., Wiswell R. A., \& Hawking, S. A. (2004). Endurance training delays age of decline in leg strength and muscle morphology. Medicine Science in Sports and Exercise, 36, 74-78.

Taunton, J. E., Ryan, M. B., \& Clement, D. B. (2002). A retrospective case-control analysis of 2002 running injuries. British Journal of Sports Medicine, 36, 95-101.

Turvey, M. T. (1990). Coordination. American psychologist, 45(8), 938.

van Emmerik, R. E. A., \& Wagenaar, R. C. (1996) Effects of walking velocity on relative phase dynamics in the trunk in human walking. Journal of Biomechanics, 29(9), 1175-84.

van Emmerik, R. E., Ducharme, S. W., Amado, A. C., \& Hamill J. (2016). Comparing dynamical systems concepts and techniques for biomechanical analysis. Journal of Sport and Health Science, $5(1), 3-13$.

van Emmerik, R. E., Hamill, J., \& McDermott, W. J. (2005). Variability and coordinative function in human gait. Quest, 57(1); 102-123. DOI:10.1080/00336297.2005.10491845

Walker, T. J. (1969). Acoustic synchrony: two mechanisms in the snowy tree cricket. Science, 166, $891-4$

Wang, L-1., Gu, C-Y., Wang, I-L., Siao, S-W., \& Chen, S-T (2018). The collision forces and lowerextremity inter-joint coordination during running. Sports Biomechanics, 17, 143-156. DOI: $10.1080 / 14763141.2016 .1249938$

Williams, G. K., \& Vicinanza, D. (2017). Coordination in gait: Demonstration of a spectral approach. Journal of Sports Sciences, 16, 1-8. 
Yen, J. T., Chang, \& Y. H. (2010). Rate-dependent control strategies stabilize limb forces during human locomotion. Journal of the Royal Society Interface, 7(46), 801-810. 
Figure 1: Joint angle waveforms throughout the stance phase for the hip (black), knee (dark grey) and ankle (light grey) for M50 (left) and M57 (right).

Figure 2: Top: Average joint synchrony $\rho_{\text {group }}$ for M50 (grey) and M57 (black). Bottom: t-test analysis (SPM $\{t\})$ of differences in joint synchrony waveforms for M50 and M57.

Figure 3: Mean (sd) joint synchrony for M50 (grey) and M57 (black) during the whole stance phase (top), absorption phase (bottom left) and propulsion phase (bottom right).

Figure 4: Degree of synchronization (average degree of synchrony, $\rho$ ) for a representative participant at M50 (A) and M57 (B); unwrapped individual phases for hip (black), knee (dark grey) and ankle (light grey) compared with the Cluster Phase (dashed line) for a representative participant at M50 (C) and M57 (D). 